\title{
Identification of novel pneumolysin alleles from paediatric carriage isolates of Streptococcus pneumoniae
}

\author{
J. M. C. Jefferies, ${ }^{1,2}$ A. S. Tocheva, ${ }^{1}$ H. Rubery, ${ }^{3}$ J. Bennett, ${ }^{3}$ J. Garland, ${ }^{3}$ \\ M. Christodoulides, ${ }^{1}$ S. N. Faust, ${ }^{1,3}$ A. Smith, ${ }^{4}$ T. J. Mitchell ${ }^{5}$ \\ and S. C. Clarke ${ }^{1,6}$
}

Correspondence

J. M. C. Jefferies

j.jefferies@southampton.ac.uk

Received 4 January 2010

Accepted 18 March 2010

\author{
${ }^{1}$ Molecular Microbiology \& Infection, Division of Infection, Inflammation and Immunity, University of \\ Southampton School of Medicine, Southampton General Hospital, Southampton SO16 6YD, UK \\ ${ }^{2}$ Health Protection Agency South East Regional Microbiology Laboratory, Southampton University \\ Hospitals NHS Trust, Southampton SO16 6YD, UK \\ ${ }^{3}$ Wellcome Trust Clinical Research Facility, University of Southampton, Southampton University \\ Hospitals NHS Trust, Southampton SO16 6YD, UK \\ ${ }^{4}$ Infection Research Group, Glasgow Dental Hospital \& School, Glasgow G2 3JZ, UK \\ ${ }^{5}$ Division of Infection \& Immunity, Glasgow Biomedical Research Centre, University of Glasgow, \\ Glasgow G12 8TA, UK \\ ${ }^{6}$ Hampshire \& Isle of Wight Health Protection Unit, Fusion 2, Parkway, Solent Business Park, \\ Whiteley P015 7AB, UK
}

\begin{abstract}
Pneumolysin (Ply) is a major virulence factor of Streptococcus pneumoniae and is produced by all known clinical isolates of pneumococci. Pneumolysin toxoids are being considered as vaccine candidates. We investigated the diversity of pneumolysin among 194 nasopharyngeal pneumococci characterized by serotyping and multilocus sequence typing (MLST). Eight Ply protein alleles were identified, four of which were novel. The 4 novel alleles varied at 10 different amino acid positions, from a total of 147, 3 of these substitutions have been previously reported in different combinations. The protein allele correlated closely with MLST. It is critical that the presence of pneumolysin variants is considered with regards to the potential use of Ply in future vaccine formulations, as variation in Ply amino acid sequence may influence the immunogenicity of vaccines based on the presence of an individual Ply allele.
\end{abstract}

\section{INTRODUCTION}

Streptococcus pneumoniae is a major cause of mortality and morbidity worldwide. Pneumococci are considered opportunistic pathogens as they are commonly carried asymptomatically in the nasopharynx of young children (Bogaert et al., 2004; Garcia-Rodriguez \& Fresnadillo Martinez, 2002). However, in a minority of cases the organism can go on to cause respiratory disease and childhood otitis media, or invasive disease such as septicaemia and meningitis (Gray et al., 1980).

Pneumolysin (Ply) is a major virulence factor of $S$. pneumoniae and belongs to the family of cholesterol-

Abbreviations: gDNA, genomic DNA; MLST, multilocus sequence typing; ST, sequence type.

The GenBank/EMBL/DDBJ accession numbers for the ply gene sequences of $S$. pneumoniae reported in this work are GU968217 to GU968411. dependent cytolysins (Jedrzejas, 2001). The family consists of $50-60 \mathrm{kDa}$ single-chain proteins produced by at least seven Gram-positive bacteria from the genera Streptococcus, Bacillus, Clostridium, Listeria and Arcanobacterium (Alouf et al., 1984). Ply is a $53 \mathrm{kDa}$ pore-forming toxin and as such has lytic effects on many mammalian cell types, and at sublytic concentrations Ply has numerous other effects, including complement activation in the absence of antipneumolysin antibodies and induction of pro-inflammatory mediators (Hirst et al., 2000; Mitchell \& Andrew, 1997; Paton et al., 1984; Zysk et al., 2001). Complement activation is independent of the haemolytic activity of the toxin (Berry et al., 1995). Pneumolysin is now known to interact directly with Toll-like receptor 4 (TLR-4) and signal via myeloid differentiation marker 88 to induce production of tumour necrosis factor alpha and interleukin 6 (Malley et al., 2003; Trzcinski et al., 2008). Pneumolysin is produced by all known clinical isolates of $S$. pneumoniae regardless of serotype and genotype. Pneumolysin toxoids 
are being considered as vaccine candidates, either alone or as a carrier protein for the pneumococcal polysaccharides used in current vaccines. The amino acid sequence of pneumolysin was previously thought to be well conserved (Mitchell et al., 1990), but we and others have reported the existence of at least 16 different pneumolysin variants at the protein level (Jefferies et al., 2007b; Kirkham et al., 2006a; Lock et al., 1996). Importantly, a number of these variants lack haemolytic activity. All S. pneumoniae isolates in these previous studies were from patients with invasive disease. We have previously hypothesized that non-haemolytic pneumolysin may be associated with an increased ability for host colonization (Kirkham et al., 2006a). The rationale for the current study was to investigate the diversity and distribution of the pneumolysin alleles present in pneumococci isolated from nasopharyngeal carriage and determine whether there was any correlation with observed differences in haemolytic activity.

\section{METHODS}

Bacterial isolates. Nasophayngeal swabbing of children less than 4 years of age attending the Paediatric Outpatient Department of Southampton University Hospitals NHS Trust, Southampton, UK, was performed according to World Health Organization guidelines (O’Brien \& Nohynek, 2003) using a cotton-tipped wire swab. Swabbing occurred with two periods during and 1 year after the introduction of the heptavalent conjugate vaccine (PCV7) into the UK childhood vaccination schedule (October 2006 to February 2007, $n=93$; and November 2007 to February 2008, $n=101$ ). Sampling was performed as part of a wider study designed to characterize colonizing pneumococcal serotypes and clones in subsequent years following the introduction of PCV7, and as such was a population snapshot. The vaccination status of children was not known but was assumed to be low in the first year, and in both years it was assumed that any herd immunity effect would be low. Swabs were anonymized and placed immediately into transport medium. Ethical approval for collection of nasopharyngeal S. pneumoniae was given by the Southampton \& South West Hampshire Research Ethics Committee (B); (Research Ethics Committee no. 06/Q1704/105), and was given research and development approval by the University of Southampton (RHM MED0704). Swabs were plated onto selective medium on the same day and plates were incubated for $18 \mathrm{~h}$ at $37{ }^{\circ} \mathrm{C}, 5 \% \mathrm{CO}_{2}$, and examined for colonies of $S$. pneumoniae. Individual colonies were subcultured onto fresh Columbia blood agar plates (E \& $\mathrm{O}$ Laboratories) and stored on beads at $-80{ }^{\circ} \mathrm{C}$.

DNA preparation and sequencing. Frozen S. pneumoniae isolates were streaked onto blood agar plates and incubated at $37{ }^{\circ} \mathrm{C}, 5 \%(\mathrm{v} / \mathrm{v})$ $\mathrm{CO}_{2}$, for $18 \mathrm{~h}$. Single colonies were suspended in $200 \mu \mathrm{l}$ lysis buffer [10 mM Tris, $100 \mathrm{mM} \mathrm{EDTA,} 0.5 \%$ (w/v) SDS] and incubated at $37{ }^{\circ} \mathrm{C}$ for $1 \mathrm{~h}$. Genomic DNA (gDNA) was prepared from this lysate using a QiaAmp DNA kit (Qiagen). The quality of the gDNA was assessed by PCR using primers $27 \mathrm{R}$ and $27 \mathrm{~S}$ as described previously (Kirkham et al., 2006a). ply gene sequencing was performed by Qiagen Genomic Services using primers and conditions as described previously (Jefferies et al., 2007b; Kirkham et al., 2006a). All primers are listed in Table 1. All ply gene sequences generated in this study have been deposited in GenBank under accession numbers GU968217 to GU968411.

Serotyping and multilocus sequence typing (MLST). gDNA samples as prepared above were used to determine serotype and multilocus sequence type (ST). Multiplex PCR was used to determine
Table 1. Primers used for PCR and sequencing of the ply gene

\begin{tabular}{|ll|}
\hline $\begin{array}{l}\text { Primer } \\
\text { name }\end{array}$ & \multicolumn{1}{c|}{ Sequence $\left(\mathbf{5}^{\prime} \rightarrow \mathbf{3}^{\prime}\right)$} \\
\hline $4 \mathrm{~V}$ & CAATACAGAAGTGAAGGCGG \\
$4 \mathrm{~T}$ & GTTGATCGTGCTCCGATGAC \\
$4 \mathrm{~W}$ & GATCATCAAGGTAAGGAAGTC \\
$27 \mathrm{R}$ & CTTGGCTACGATATTGGC \\
$27 \mathrm{~S}$ & TACTTAGTCCAACCACGG \\
$27 \mathrm{~T}$ & ATAAGTCATCGGAGCACG \\
$9 \mathrm{Y}$ & CGGGATCCGGCAAATAAAGCAGTAAATGACTTT \\
$9 \mathrm{Z}$ & GACGGAGCTCGACTAGTCATTTTCTACCTTATC \\
\hline
\end{tabular}

the serogroup/serotype of each isolate according to the method of Pai et al. (2006). We also made use of the Centers for Disease Control Streptococcal Laboratory $S$. pneumoniae capsular serotyping methodology website (www.cdc.gov/ncidod/biotech/strep/pcr.htm), which periodically posts updates to the above method. MLST was performed by Qiagen Genomic Services using the method of Enright \& Spratt (1998) with use of the MLST website (www.mlst.net). Analysis of relationships between STs was carried out using the program eBURST-2 (Feil et al., 2004), which can be accessed at www.mlst.net.

Crude haemolytic assays of lysates of carriage S. pneumoniae. Pneumococcal strains were subcultured from frozen stocks onto Columbia blood agar plates, incubated at $37{ }^{\circ} \mathrm{C}, 5 \%(\mathrm{v} / \mathrm{v}) \mathrm{CO}_{2}$, and a single colony was inoculated into $10 \mathrm{ml}$ brain heart infusion (BHI) medium and incubated overnight. From this culture, $2 \mathrm{ml}$ was inoculated into $18 \mathrm{ml}$ pre-warmed $\mathrm{BHI}$ and grown to exponential phase. The culture was centrifuged and the supernatant decanted, with the resulting pellet then resuspended in $1 \mathrm{ml}$ PBS and lysed by sonication using a probe sonicator (Soniprep 150; MSE). The protein concentration of the lysate was quantified using a BCA protein assay kit (Pierce) and sample concentrations were adjusted to $400 \mu \mathrm{g}$ protein $\mathrm{ml}^{-1}$. Lysate $(100 \mu \mathrm{l})$ was then used to prepare a dilution series with which to assess haemolytic activity with a $2 \%(\mathrm{v} / \mathrm{v})$ suspension of sheep red blood cells. The end point of the assay was measured as the point at which a 'button' of non-lysed red blood cells could be visualized. $S$. pneumoniae strains D39 and 00-3645 were used as positive and negative controls, respectively, for haemolysis (Kirkham et al., 2006a).

\section{RESULTS}

\section{Carriage isolates}

A total of 194 S. pneumoniae were isolated and represented the total number of swabs culture-positive for S. pneumoniae in each study period; the carriage rate in each year was 29.6 and $27.8 \%$, for October 2006-February 2007 and November 2007-February 2008, respectively.

\section{Four novel Ply protein alleles were identified from pneumococcal carriage isolates}

Bacterial DNA sequences were aligned using AlignX (VectorNTI; Invitrogen). DNA sequences were translated into amino acid sequences and a second alignment was performed, which resulted in the identification of eight different Ply alleles at the protein level, of which four had 
not been previously identified. Ply alleles $1(n=79)$ and 2 $(n=86)$ were the most common types of Ply protein present among the 194 carriage isolates, mirroring the observations made regarding Ply distribution in invasive pneumococcal isolates (Jefferies et al., 2007b; Kirkham et al., 2006a; Lock et al., 1996). A Ply identical to the allele previously reported as allele 8 (Jefferies et al., 2007b) was observed for ten strains and a further seven strains possessed an allele identical to that previously called allele 9 in a study of pneumolysin diversity among S. pneumoniae isolated from invasive disease (Jefferies et al., 2007b). The remaining 12 strains possessed 1 of 4 novel Ply alleles, which we have referred to as alleles 15 to 18 . These four novel alleles contained amino acid substitutions at a total of ten positions, three of which have been reported previously (Jefferies et al., 2007b). Ply allele 15 was present in nine strains whereas Ply alleles 16-18 were observed for single isolates only. Table 2 depicts amino acid changes present among the eight alleles identified in this study and compares these alleles to other known Ply alleles.

\section{Distribution of Ply alleles among carriage S. pneumoniae is associated with clonal type}

Relationships between STs of the isolates included in this study were examined using eBURST and the distribution of Ply alleles plotted (Fig. 1). The distribution of Ply alleles in this collection of carriage isolates appears to be clonal, that is to say the Ply allele is more closely associated with ST than with serotype. However, Ply alleles 1 and 2 are observed in a wider range of genetic backgrounds and serotypes than are the other observed alleles. Ply allele 1 was observed in 78 pneumococcal strains of serotypes $23 \mathrm{~F}$, 19F and serogroup 6, representing 29 different STs. Ply allele 2 was present in 87 isolates of serotypes 3, 35F, 4, $14,34,19 \mathrm{~A}, 11 \mathrm{~A}, 16 \mathrm{~F}, 17 \mathrm{~F}$ and 38 and serogroup 6 representing 27 different STs. Allele 8 was identified in ten isolates, all of which belonged to ST36; all except one of these were serotype $23 \mathrm{~F}$, whilst one was a serotype 11 isolate of the same genotype. In a previous study of Ply allele distribution among invasive pneumococcal isolates Ply allele 8 was observed in a single invasive serotype 23F, ST40 isolate (Jefferies et al., 2007b). ST40 is a double locus variant and so part of the same clonal group as ST36, observed here carrying allele 8 . Allele 9 was identified in seven carriage isolates, all of which were ST162 serotype 19F isolates. Ply allele 9 has previously been observed in this background (Jefferies et al., 2007b).

Of the newly identified Ply alleles (alleles 15-18), alleles 16, 17 and 18 were observed in single isolates only, whilst allele 15 was present in nine isolates, seven of which were serotype $19 \mathrm{~F}$ and belonged to ST179 or its single locus variant ST177. Ply allele 15 differs from allele 2 at only 1 amino acid position (E260D); this substitution has been previously observed in allele 13, although that allele contains $\mathrm{N}$ at position 380, whilst allele 15 contains $\mathrm{D}$. The remaining isolate was a serogroup 6 isolate with unrelated ST, ST398. Novel Ply alleles 16, 17 and 18 were observed in single isolates of serogroup 6, an acapsular isolate and serotype 11A. Alleles 16, 17 and 18 contained previously unreported amino acid substitutions at positions 296, 323, 386, 394, 439 and 468 (Table 2). It should be noted that isolate 2327 containing Ply allele 17 may be an atypical mitis-group Streptococcus species rather than a true pneumococcal isolate as it contains seven new MLST alleles that when concatenated do not align well with a database of concatenated pneumococcal MLST allele sequences (www.mlst.net). Although isolate 2327 was optochin sensitive, it was negative for $c p s A$ by PCR using the primer pair published by Pai et al. (2006). Pneumolysin-like alleles from such isolates have been reported in the past (Jefferies et al., 2007a; Whatmore et al., 2000). Allele 17 differs at 4 amino acid positions from allele 1 . We have previously observed two of these substitutions (N14D and K224R) in both pneumolysin and mitilysin, a closely related protein found in certain $S$. mitis isolates (Jefferies et al., 2007a). The remaining two substitutions (D323N and $\mathrm{H} 386 \mathrm{~N}$ ) are novel. It should be noted that although no cpsA band could be amplified from this particular isolate, in general a cpsA negative result should not necessarily be interpreted as meaning the isolate is negative for cpsA as approximately $1-2 \%$ of PCR serotypable pneumococci test cpsA negative (www.cdc. gov/ncidod/biotech/strep/pcr.htm). In this case, the MLST result together with the Ply allele and lack of cpsA suggest an atypical mitis-group Streptococcus.

S. pneumoniae isolates 110, 160, 163, 165, 221, 2029, 2327 and 2329 were chosen as representatives of the eight Ply protein alleles observed in our study. Haemolytic activity was observed for lysates of all eight strains (Table 3). We observed differences in haemolytic activity of up to 31 -fold between isolates. These values should be interpreted with caution and may not reflect true differences in Ply activity; our assay was carried out on crude pneumococcal lysates only and differences in the haemolytic activity observed could be due to differential expression of pneumolysin, and may also be affected by other cellular factors present in the lysate.

\section{DISCUSSION}

This is believed to be the first study of the allelic distribution of the cholesterol-dependent cytolysin pneumolysin amongst $S$. pneumoniae isolated from asymptomatic nasopharyngeal carriage. We report the presence of 8 pneumolysin alleles from a collection of 198 isolates, 4 of which were novel allelic variants. Analysis of the distribution of Ply alleles amongst these carriage isolates revealed that variants of pneumolysin are clonally distributed, and strengthens evidence previously collected from invasive S. pneumoniae (Jefferies et al., 2007b) in support of this hypothesis, and showed that variation in Ply occurs in S. pneumoniae associated with carriage and with invasive disease. Recent studies have shown the clonal distribution of a number of pneumococcal proteins 
Table 2. Pneumolysin alleles showing amino acid positions at which variation occurs

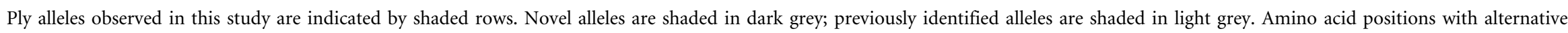
residues identified in this study are shown in italics. INS, 7 amino acid insertion; DEL, single amino acid deletion.

\begin{tabular}{|c|c|c|c|c|c|c|c|c|c|c|c|c|c|c|c|c|c|c|c|c|c|c|}
\hline \multirow[t]{2}{*}{ Allele } & \multicolumn{22}{|c|}{ Amino acid and its position } \\
\hline & $14 N$ & $136 Q$ & 142 & $150 Y$ & $154 \mathrm{~T}$ & $167 S$ & $172 \mathrm{~T}$ & $224 K$ & $260 \mathrm{E}$ & $265 A$ & 267I & $270 \mathrm{~V}$ & $271 \mathrm{~K}$ & $273 A$ & $296 \mathrm{P}$ & 323D & $380 \mathrm{D}$ & $386 \mathrm{H}$ & $394 \mathrm{P}$ & $402 Q$ & 415 & $439 \mathrm{~V} 468 \mathrm{~V}$ \\
\hline 1 & & & & & & & & & & & & & & & & & & & & & & \\
\hline 2 & & & & & & & & & & & & & & & & & $\mathrm{~N}$ & & & & & \\
\hline 3 & & & & & & & I & $\mathrm{R}$ & & $S$ & & DEL & DEL & & & & & & & & & \\
\hline 4 & & & & & & & & & & & & & & & & & $\mathrm{~N}$ & & & & INS & \\
\hline 5 & & & & $\mathrm{H}$ & & & I & $\mathrm{R}$ & & S & & DEL & DEL & & & & & & & & & \\
\hline 6 & & & & & & $\mathrm{~F}$ & I & $\mathrm{R}$ & & S & & DEL & DEL & & & & & & & & & \\
\hline 7 & $\mathrm{D}$ & & & & & & & $\mathrm{R}$ & & & & & & & & & & & & & & \\
\hline 8 & & & & & & & & & & & & & & $\mathrm{D}$ & & & & & & & & \\
\hline 9 & & & & & & & & & & & M & & & & & & & & & & & \\
\hline 10 & & & & M & & & & & & $S$ & & DEL & DEL & & & & $\mathrm{N}$ & & & & & \\
\hline 11 & & $\mathrm{~K}$ & & & & & & & & & & & & & & & $\mathrm{~N}$ & & & & & \\
\hline 12 & & & & & & & & & & & & & & & & & $\mathrm{~N}$ & & & E & & \\
\hline 13 & & & & & & & & & $\mathrm{D}$ & & & & & & & & $\mathrm{N}$ & & & & & \\
\hline 14 & & & INS & & & & & & & & & & & & & & & & & & & \\
\hline 15 & & & & & & & & & $\mathrm{D}$ & & & & & & & & & & & & & \\
\hline 16 & & & & & & & & $\mathrm{R}$ & & & & & & & $S$ & & & & $A$ & & & $I$ \\
\hline 17 & $\mathrm{D}$ & & & & & & & $\mathrm{R}$ & & & & & & & & $\mathrm{N}$ & & $\mathrm{N}$ & & & & \\
\hline 18 & & & & & & & & & & & & & & & & & & & & & & $I$ \\
\hline
\end{tabular}




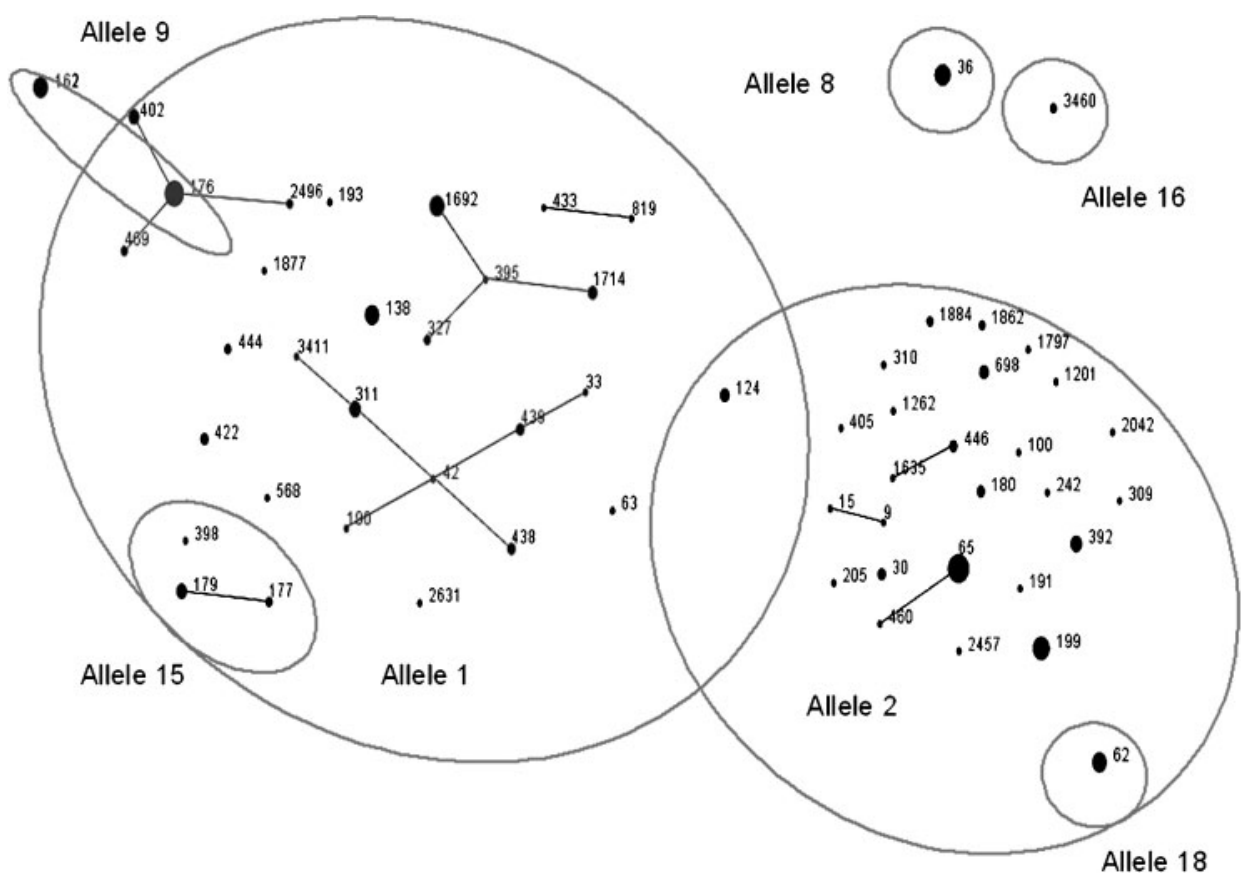

Fig. 1. eBURST analysis of the clonal relationships between S. pneumoniae showing the distribution of pneumolysin alleles. The STs encoding the 7 Ply alleles observed in this study are shown contained within circles (allele 17 was not included in this analysis as it could not be assigned an ST).

involved in adherence and pathogenesis, such as pilus and pneumococcal surface protein PclA (Paterson et al., 2008; Aguiar et al., 2008; Moschioni et al., 2008). Other virulence factors, such as neuraminidase, are also associated with clonal type; however, such relationships may be more difficult to assess as there is a higher level of intraclonal variation (Pettigrew et al., 2006).

A recent study by Dagerhamn et al. (2008) has shown that there is less intraclonal variation in accessory genes among STs representative of serotypes with high invasive disease potential than among STs associated with carriage. Here we show that there is little intraclonal variation of pneumolysin within STs of carriage S. pneumoniae but that interclonal variation is marked. Moreover, we did not observe non-haemolytic Ply alleles, such as that previously reported as allele 5 , in any of the $S$. pneumoniae isolated from carriage in this study. This is probably due to the fact that allele 5 is associated with clonal complexes related to ST306 and ST53, and neither of these clonal groups was represented in the carriage isolate collection used in this study. These clones are representatives of serotypes 1 and 8 ,

Table 3. Haemolytic activity of crude lysates of representative pneumococcal strains

Lysates were adjusted to $400 \mu \mathrm{g}$ total protein $\mathrm{ml}^{-1}$.

\begin{tabular}{|lcccc|}
\hline Strain & ST & Serotype & Ply allele & $\begin{array}{c}\text { Haemolytic activity } \\
\text { [HU (mg total protein) }\end{array}$ \\
\hline 110 & 311 ] \\
163 & 205 & $23 \mathrm{~F}$ & 1 & 0.08 \\
2329 & 62 & $11 \mathrm{~A}$ & 2 & 1.25 \\
221 & 162 & $19 \mathrm{~F}$ & 8 & 0.04 \\
165 & 177 & $19 \mathrm{~F}$ & 9 & 0.16 \\
2029 & 3460 & $6 \mathrm{~B}$ & 16 & 0.08 \\
$2327^{*}$ & 7 new alleles & $\mathrm{N} / \mathrm{T}$ & 17 & 0.63 \\
160 & 62 & $11 \mathrm{~A}$ & 18 & 0.63 \\
& & & & 0.08 \\
\hline
\end{tabular}

HU, Haemolytic unit.

${ }^{\star}$ Strain 2327 is optochin sensitive but MLST suggests that this strain is an atypical mitis-group Streptococcus. 
which are rarely isolated from paediatric carriage studies. The four novel Ply alleles observed in this study were all haemolytic, this is in accordance with previous findings as none of these alleles contained mutations of residue Y150, which has been shown to be absolutely necessary for haemolytic activity (Kirkham et al., 2006a, b).

We have shown that Ply distribution among S. pneumoniae varies with ST and that Ply alleles present in carriage strains are similar to those found among invasive isolates, although no non-haemolytic alleles were observed among the carriage isolates in this study. Our finding is in agreement with the hypothesis that a predilection for either carriage or invasive disease among pneumococcal isolates is associated with genetic background and gene expression (i.e. a collection of various genes and gene products working in concert) rather than being linked to the presence or absence of certain virulence factors or specific alleles thereof. Variations in Ply have been associated with lack of reactivity to mAb (Jefferies et al., 2007a), which could lead to false-positive results if such antibodies are used in diagnostic tests for $S$. pneumoniae. It is critical that the presence of pneumolysin variants is taken into account when considering the potential use of this protein in future vaccine formulations, as variation in Ply amino acid sequence may influence the immunogenicity of vaccines based on the presence of an individual Ply allele.

\section{ACKNOWLEDGEMENTS}

This work makes use of the MLST website (www.mlst.net). We thank the staff of the HPA (Health Protection Agency) South East Regional Microbiology Laboratory, Southampton, for isolation of pneumococci. This work was funded by a pump-priming grant from the UK HPA.

\section{REFERENCES}

Aguiar, S. I., Serrano, I., Pinto, F. R., Melo-Cristino, J. \& Ramirez, M. (2008). The presence of the pilus locus is a clonal property among pneumococcal invasive isolates. BMC Microbiol 8, 41.

Alouf, J. E., Geoffroy, C., Pattus, F. \& Verger, R. (1984). Surface properties of bacterial sulfhydryl-activated cytolytic toxins. Interaction with monomolecular films of phosphatidylcholine and various sterols. Eur J Biochem 141, 205-210.

Berry, A. M., Alexander, J. E., Mitchell, T. J., Andrew, P. W., Hansman, D. \& Paton, J. C. (1995). Effect of defined point mutations in the pneumolysin gene on the virulence of Streptococcus pneumoniae. Infect Immun 63, 1969-1974.

Bogaert, D., De Groot, R. \& Hermans, P. W. (2004). Streptococcus pneumoniae colonisation: the key to pneumococcal disease. Lancet Infect Dis 4, 144-154.

Dagerhamn, J., Blomberg, C., Browall, S., Sjostrom, K., Morfeldt, E. \& Henriques-Normark, B. (2008). Determination of accessory gene patterns predicts the same relatedness among strains of Streptococcus pneumoniae as sequencing of housekeeping genes does and represents a novel approach in molecular epidemiology. J Clin Microbiol 46, 863-868.

Enright, M. C. \& Spratt, B. G. (1998). A multilocus sequence typing scheme for Streptococcus pneumoniae: identification of clones associated with serious invasive disease. Microbiology 144, 30493060.

Feil, E. J., Li, B. C., Aanensen, D. M., Hanage, W. P. \& Spratt, B. G. (2004). eBURST: inferring patterns of evolutionary descent among clusters of related bacterial genotypes from multilocus sequence typing data. J Bacteriol 186, 1518-1530.

Garcia-Rodriguez, J. A. \& Fresnadillo Martinez, M. J. (2002). Dynamics of nasopharyngeal colonization by potential respiratory pathogens. J Antimicrob Chemother 50, 59-74.

Gray, B. M., Converse, G. M., III \& Dillon, H. C., Jr (1980). Epidemiologic studies of Streptococcus pneumoniae in infants: acquisition, carriage, and infection during the first 24 months of life. J Infect Dis 142, 923-933.

Hirst, R. A., Rutman, A., Sikand, K., Andrew, P. W., Mitchell, T. J. \& O'Callaghan, C. (2000). Effect of pneumolysin on rat brain ciliary function: comparison of brain slices with cultured ependymal cells. Pediatr Res 47, 381-384.

Jedrzejas, M. J. (2001). Pneumococcal virulence factors: structure and function. Microbiol Mol Biol Rev 65, 187-207.

Jefferies, J., Nieminen, L., Kirkham, L. A., Johnston, C., Smith, A. \& Mitchell, T. J. (2007a). Identification of a secreted cholesteroldependent cytolysin (mitilysin) from Streptococcus mitis. J Bacteriol 189, 627-632.

Jefferies, J. M., Johnston, C. H., Kirkham, L. A., Cowan, G. J., Ross, K. S., Smith, A., Clarke, S. C., Brueggemann, A. B., George, R. C. \& other authors (2007b). Presence of nonhemolytic pneumolysin in serotypes of Streptococcus pneumoniae associated with disease outbreaks. J Infect Dis 196, 936-944.

Kirkham, L. A., Jefferies, J. M., Kerr, A. R., Jing, Y., Clarke, S. C., Smith, A. \& Mitchell, T. J. (2006a). Identification of invasive serotype 1 pneumococcal isolates that express nonhemolytic pneumolysin. J Clin Microbiol 44, 151-159.

Kirkham, L. A., Kerr, A. R., Douce, G. R., Paterson, G. K., Dilts, D. A., Liu, D. F. \& Mitchell, T. J. (2006b). Construction and immunological characterization of a novel nontoxic protective pneumolysin mutant for use in future pneumococcal vaccines. Infect Immun 74, 586-593.

Lock, R. A., Zhang, Q. Y., Berry, A. M. \& Paton, J. C. (1996). Sequence variation in the Streptococcus pneumoniae pneumolysin gene affecting haemolytic activity and electrophoretic mobility of the toxin. Microb Pathog 21, 71-83.

Malley, R., Henneke, P., Morse, S. C., Cieslewicz, M. J., Lipsitch, M., Thompson, C. M., Kurt-Jones, E., Paton, J. C., Wessels, M. R. \& Golenbock, D. T. (2003). Recognition of pneumolysin by Toll-like receptor 4 confers resistance to pneumococcal infection. Proc Natl Acad Sci U S A 100, 1966-1971.

Mitchell, T. J. \& Andrew, P. W. (1997). Biological properties of pneumolysin. Microb Drug Resist 3, 19-26.

Mitchell, T. J., Mendez, F., Paton, J. C., Andrew, P. W. \& Boulnois, G. J. (1990). Comparison of pneumolysin genes and proteins from Streptococcus pneumoniae types 1 and 2. Nucleic Acids Res 18, 4010.

Moschioni, M., Donati, C., Muzzi, A., Masignani, V., Censini, S., Hanage, W. P., Bishop, C. J., Reis, J. N., Normark, S. \& other authors (2008). Streptococcus pneumoniae contains $3 \mathrm{rlr}$ A pilus variants that are clonally related. J Infect Dis 197, 888-896.

O'Brien, K. L., Nohynek, H. \& the World Health Organization Pneumococcal Vaccine Trials Carriage Working Group (2003). Report from a WHO working group: standard method for detecting upper respiratory carriage of Streptococcus pneumoniae. Pediatr Infect Dis J 22, 133-140.

Pai, R., Gertz, R. E. \& Beall, B. (2006). Sequential multiplex PCR approach for determining capsular serotypes of Streptococcus pneumoniae isolates. J Clin Microbiol 44, 124-131. 
Paterson, G. K., Nieminen, L., Jefferies, J. M. \& Mitchell, T. J. (2008). PclA, a pneumococcal collagen-like protein with selected strain distribution, contributes to adherence and invasion of host cells. FEMS Microbiol Lett 285, 170-176.

Paton, J. C., Rowan-Kelly, B. \& Ferrante, A. (1984). Activation of human complement by the pneumococcal toxin pneumolysin. Infect Immun 43, 1085-1087.

Pettigrew, M. M., Fennie, K. P., York, M. P., Daniels, J. \& Ghaffar, F. (2006). Variation in the presence of neuraminidase genes among Streptococcus pneumoniae isolates with identical sequence types. Infect Immun 74, 3360-3365.

Trzcinski, K., Thompson, C. M., Srivastava, A., Basset, A., Malley, R. \& Lipsitch, M. (2008). Protection against nasopharyngeal colonization by Streptococcus pneumoniae is mediated by antigen-specific $\mathrm{CD} 4^{+} \mathrm{T}$ cells. Infect Immun 76, 2678-2684.

Whatmore, A. M., Efstratiou, A., Pickerill, A. P., Broughton, K., Woodard, G., Sturgeon, D., George, R. \& Dowson, C. G. (2000). Genetic relationships between clinical isolates of Streptococcus pneumoniae, Streptococcus oralis, and Streptococcus mitis: characterization of "atypical" pneumococci and organisms allied to S. mitis harboring S. pneumoniae virulence factor-encoding genes. Infect Immun 68, 1374-1382.

Zysk, G., Schneider-Wald, B. K., Hwang, J. H., Bejo, L., Kim, K. S., Mitchell, T. J., Hakenbeck, R. \& Heinz, H. P. (2001). Pneumolysin is the main inducer of cytotoxicity to brain microvascular endothelial cells caused by Streptococcus pneumoniae. Infect Immun 69, 845-852. 